and a systematic search was carried out specifically for identification of deleterious genetic variants in genes involved in novel inflammatory pathways.

Results: The index case was coming from a consanguineous family of Assyrian origin, who is now 22-year-old male. He presented to our outpatient clinic with recurrent attacks of fever, urticarial rash on the extremities and trunk, conjunctival injections and arthralgia, without a trigger or more frequently following an infection. His attacks started when he was 13 , and two to three days lasting attacks recurred more frequently during warm weather conditions or following hot baths. He had highly elevated CRP and ESR during attacks, but his acute phase response did not return to normal values in between the flares. Low C3 and C4 values were also observed during asymptomatic periods. His ANA test became positive during the course of his disease, with an increasing titer in the last year. He responded partially to corticosteroids as well as canakinumab and anakinra treatments, and he is currently on low dose steroids and 100 to $200 \mathrm{mg} /$ day anakinra. Whole exome sequencing revealed a deleterious homozygous c.769C>T mutation in AGBL3 (ATP/GTP binding protein-like 3) gene, which results in early termination of the protein (p.GIn257Ter) and deletion of the functional carboxypeptidase domain. This protein belongs to metallocarboxypeptidases that mediate both deglutamylation and deaspartylation of target proteins. AGBL3 is suggested to catalyse the deglutamylation of polyglutamate side chains, especially in proteins such as tubulins. Also, STRING search revealed the interaction of AGBL3 with complement regulatory proteins, such as CD46, CD55, and CD59, which are potent inhibitors of the complement membrane attack complex. We searched databases from Turkey and other sources including 1000 Genomes Project data, and we could not identify this variant in other individuals.

Conclusions: This study identifies the AGBL3 metallocarboxypeptidase gene as a potential autoinflammatory gene involved in a novel pathway and possibly associated with hypocomplementemic urticarial vasculitis phenotype. Previously, DNASE1L3 mutations have been associated with hypocomplementemic urticarial vasculitis and systemic lupus erythematosus phenotype. The loss of function mutation in the AGBL3 may result in a potent innate inflammatory response as well as autoimmunity through a new pathway, which is resulting in lower complement levels and ANA positivity along with recurrent inflammatory episodes. This complex phenotype explains a partial response to the IL-1 blockade, and further studies in patients/families with a similar phenotype are needed.

Disclosure of Interest: None declared

DOI: 10.1136/annrheumdis-2018-eular.6490

\section{SAT0598 SYSTEMATIC LITERATURE REVIEW ON THE EFFICACY AND SAFETY OF IMMUNOMODULATORY DRUGS IN PATIENTS WITH NONIFECTIOUS INTERMEDIATE AND POSTERIOR UVEITIS, PANUVEITIS AND MACULAR OEDEMA}

A. Gómez Gómez ${ }^{1,2}$, E. Loza ${ }^{3}$, M.P. Rosario ${ }^{3}$, G. Espinosa ${ }^{4}$, J. M$^{5}$, J.M. Herreras $^{6}$, S. Muñoz ${ }^{7,8}$, M. Cordero-Coma ${ }^{9} .{ }^{1}$ Rheumatology, Hospital Infanta Sofía; ${ }^{2}$ Universidad Complutense de Madrid; ${ }^{3}$ Instituto de Salud Musculoesquelética (INMUSC), Madrid; ${ }^{4}$ Department of Autoimmune Diseses, Institut Clinic de Medicina i Dermatologia, Hospital Clínic., Barcelona; ${ }^{5}$ Unidad de Inmunología, Complejo Asistencial Universitario e Instituto de Biomedicina Universidad de León (IBIOMED), León; ${ }^{6}$ IOBA, Universidad de Valladolid, CIBER-BBN, Hospital Clínico Universitario de Valladolid, Valladolid; ${ }^{7}$ Universidad Europea de Madrid; ${ }^{8}$ Rheumatology, Hospital Universitario Infanta Sofía, Madrid; ${ }^{9}$ Unidad de Uveitis, Complejo Asistencial Universitario e Instituto de Biomedicina Universidad de León (IBIOMED), León, Spain

Background: Posterior segment uveitis (PSU) is a sight-threatening condition Objectives: To perform a systematic review of the literature on the use of immunomodulatory drugs in adult patients with non-infectious and non-malignant PSU including intermediate (IU) and posterior uveitis (PU), panuveitis (PanU) and macular oedema (ME)

Methods: Search strategies were designed for Medline, Embase, and Cochrane Library for articles with clinical, safety or cost-effectivity data up to, Jan 2017 followed by secondary search from their bibliography. Quality was assessed (Jadad/ Oxford)

Results: From 1103 articles, 31 moderate quality clinical trials (CT) were selected, prospective/retrospective, with variability in mean duration, No. and patients'characteristics. PSU was treated with synthetic DMARDs methotrexate (MTX), azathioprine (AZA), cyclosporine A (CsA), cyclopshosphamide (CyC), tacrolimus, sirolimus, micophenolate (MMF) and interferon $\beta$, and biologic DMARDs ranibizumab, daclizumab, rituximab (RTX), secukinumab, adalimumab (ADA), bevacizumab and infliximab (IFX) at usual dosages. Most common measures: visual acuity (VA), macular thickness and vitreous haze. MTX vs.MMF, was effective in IU, PU and PanU, with no differences in efficacy and adverse events (AEs), neither in Vogt-Koyanagi-Harada. MTX was effective with RTX, and SC was inferior to IFN $\beta$ with lower rate of AEs in IU with cistoid ME (CME). CsA did not show efficacy vs. placebo (pbo), with more neurological AEs. Tacrolimus vs. CsA was safer with similar efficacy, and CsA was useful with no differences vs. prednisone (pred) or vs.CsG, and similar vs.CYC at 2 y in Behçet PSU. CsA + pred + ketoconazole combined showed additional benefits. CYC+AZA were effective in PU, except for VA and retinal vasculitis, with no differences vs. RTX +MTX. CYC was useful in serpiginoid choroiditis \pm dexamethasone. ADA was useful in IU, PU and PanU vs. pbo. IFX in Behçet PSU, was more effective vs. prednisolone $+\mathrm{Cs} A+\mathrm{AZA} / \mathrm{MTX}$. Intravitreal ADA and IFX did not show any benefit. Secukinumab vs. pbo did not prevent recurrences. In another RCT, IV route showed a higher response rate vs.SC for $30 \mathrm{mg} / \mathrm{kg}$, with similar rate of EAs. Intravitreal bevacizumab was effective in multifocal choroiditis and CME. Intravitreal ranibizumab was useful in pigmentary retinitis +CME. Daclizumab in Behçet PSU did not show benefit vs. pbo. Tacrolimus as 2nd line in PU was effective \pm pred. Intravitreal and subconjunctival sirolimus were effective in IU, PU and PanU in vitreal haze but not VA and ME, improving functional scores

Studies flow chart

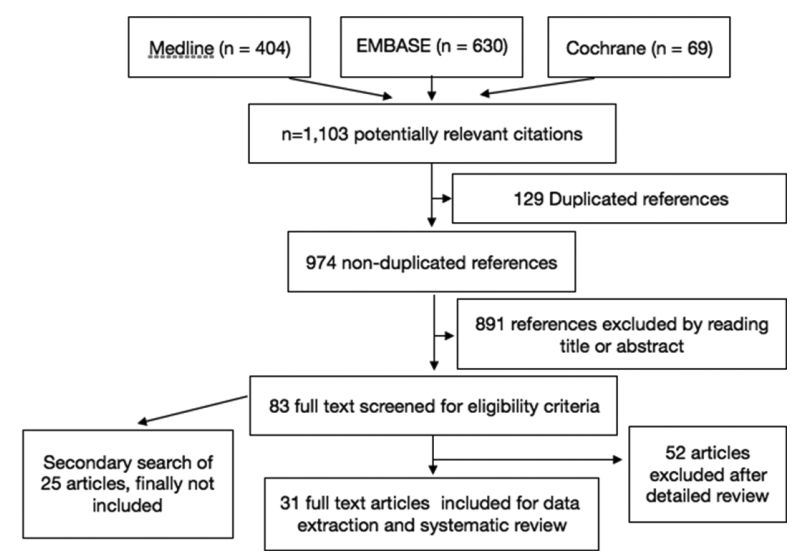

Conclusions: 1 Moderate quality of evidence 2 Variability in patients, definitions and outcomes

3 Systemic DMARDs MTX, MMF, CsA, CyC, tacrolimus, sirolimus, MMF and IFN $\beta$ were useful in PSU;AZA in combination

4 Biologic DMARDs ADA, IFX (systemic), ranibizumab, bevacizumab (intravitreal) were useful, daclizumab did not show efficacy. Possible efficacy of secukinumab 5 Intravitreal anti-TNF (ADA,IFX) were not useful

Disclosure of Interest: None declared

DOI: 10.1136/annrheumdis-2018-eular.3114

\section{SAT0599 IDIOPATHIC GRANULOMATOUS MASTITIS MAY RESPONSE WELL TO COMBINATION OF IMMUNOSUPRESIVES AND GLUCOCORTICOIDS}

A. Sari ${ }^{1}$, A. Konan ${ }^{2}$, M. Akpinar ${ }^{3}$, B. Armagan ${ }^{1}$, A. Erden ${ }^{1}$, L. Kilic ${ }^{1}$, O. Karadag ${ }^{1}$, A. Akdogan ${ }^{1}$, S. Apras Bilgen ${ }^{1}$, S. Kiraz ${ }^{1}$, I. Ertenli ${ }^{1}$, U. Kalyoncu ${ }^{1} .{ }^{1}$ Rheumatology; ${ }^{2}$ General Surgery, Hacettepe University Faculty of Medicine; ${ }^{3}$ Radiology, Hacettepe University Faculty of Medicine, Ankara, Turkey

Background: Idiopathic granulomatous mastitis (IGM) is a rare inflammatory dis ease of breast. Corticosteroids (CS) and immunosuppressive agents constitute treatment alternatives other than surgery.

Objectives: To evaluate the clinical characteristics and treatment responses of IGM patients followed up in our clinic.

Methods: The medical records of 70 IGM patients who were referred to Hacettepe University Rheumatology Clinic were examined. Forty-four patients who had at least one visit in the last 2 years were included in the analysis. Demographic, clinical and laboratory characteristics of the patients, treatments, clinical and/or ultrasonographically measured lesion sizes at the time of diagnosis and at the last follow-up were recorded. Complete and partial response in the last control visit were defined as $>50 \%$ and $<50 \%$ decrease in the lesion size, respectively. Lesions which are stable or increasing in size was accepted as unresponsive. Increase in the drug dose by the clinician or increasing of the lesion size during follow-up were considered as relapse.

Results: Median age was 35.7 (24.3-57.2) years and median symptom duration was $2.5(0.2-54.1)$ months at baseline. Palpable mass $(90.9 \%)$ and breast pain $(88.1 \%)$ were the most common symptoms. Skin fistules and axillary lymphadenopathy were present in $16(37.2 \%)$ and $15(34.1 \%)$ patients, respectively. Erythema nodosum was seen in $5(11.4 \%)$ patients during follow-up. The median follow-up duration was 10.5 (1.05-99.6) months. CS monotherapy and combination of CS and immunosuppressive were used in $3(7.8 \%)$ and 38 (86.4\%) patients, respectively. Three patients were followed up without treatment. The firs immunosuppressive agent was methotrexate (MTX) in 32 patients (84.2\%) and 